\title{
Publisher's Note: Realizing and Detecting a Topological Insulator in the AIII Symmetry Class [Phys. Rev. Lett. 119, 115301 (2017)]
}

\author{
Carlos G. Velasco and Belen Paredes
}

(Q) (Received 14 January 2019; published 25 January 2019)

DOI: 10.1103/PhysRevLett.122.039902

This paper was published online on 14 September 2017 with an omission of a support statement in the Acknowledgment section. On page 4, the Acknowledgment section should read as "The work of C. G. V. is supported through the Grant No. BES-2013-064443 of the Spanish MINECO. This research was funded by the Deutsche Forschungsgemeinschaft (DFG, German Research Foundation) via Research Unit FOR 2414 under Project No. 277974659." The Acknowledgment section has been corrected as of 15 January 2019. The Acknowledgment section is incorrect in the printed version of the journal. 